\title{
Gamut mapping for visual attention retargeting
}

\author{
Javier Vazquez-Corral and Marcelo Bertalmío; Department of Information and Communication Technologies, Universitat Pompeu \\ Fabra, E-08018, Barcelona, Spain
}

\begin{abstract}
Visual attention retargeting attempts to modify an image such that the viewer's attention is directed to specific regions. Goals include highlighting a particular object or hiding possible problems in the image. In this work, we show that we can pose the visual retargeting problem in terms of gamut mapping. In short, visual attention retargeting can be achieved by performing gamut extension in those regions that we want to highlight and gamut reduction in the other regions.
\end{abstract}

\section{Introduction}

Visual attention retargeting [8] is an emerging field of research. It traces its roots to a more general problem: image saliency estimation. Image saliency predicts the attentional gaze of observers viewing a scene. Many computational models have been defined during the recent years handling this problem, for example [1], [10], [14]. Image saliency has been also used as a cue to aid in the performance of both image processing and computer vision applications such as color to gray conversion [2], [5] or image detail visibility [12]. In short, visual attention retargeting attempts to modify the image to attract the viewer's interest to some particular region of the image. This topic is of interest for people working in advertising (focusing consumer attention to the desired areas), or cinema post-production (allowing content creators to hide errors by focusing the viewers' attention in parts of the image where the error is not present).

The seminal work on visual attention retargeting and saliency alteration was proposed by Wong and Low [15] where three low level image features, namely luminance, color saturation, and sharpness were modified to increase the saliency of a predefined region. Hagiwara et al. [6] suggested to adjust the intensity and color until the saliency inside the region of interest becomes the highest for the entire image. More recent methods are those of Mateescu and Balic [8] and Nguyen et al. [11]. These two methods obtain very good results in terms of saliency alteration, but at the cost of introducing remarkable hue shifts in the objects of interest. In particular, the work of [8] proposed a method that modifies the color of a selected region in the following way. First they represent the hue as an angle in CIELAB color space, and then they define a hue rotation as the optimal adjustment of the region of interest. This hue rotation should maximize the dissimilarity of hue distribution of the selected region relative to its surroundings. The work of Nguyen et al. [11] performs color transfer with naturalness and smoothness constraints via a Markov Random Field (MRF) framework by comparing the patches presented in the region of interest to patches from a large salient patch database. Other related works to this problem are the ones of Su et al. [13] for deemphasizing distractors in an image, Yan et al. [16] for adjusting image aesthetics and Chu et al. [4] for camouflaging objects in an image.
On a different topic, gamut mapping [9] deals with the problem of modifying the gamut of an input image to make it match with the destination gamut which varies depending on the medium used to visualize the image. The problem of gamut mapping can be therefore subdivided into gamut reduction (in the case the gamut of the input image should be reduced) and gamut extension (in the case the gamut of the original image should be extended).

The goal of this paper is to show how we can use a gamut mapping method to perform visual attention retargeting without introducing large color differences between the original and the resulting image. Our idea builds on the hypothesis that saliency regions are, in general, those that are closer to the boundary of the gamut. Therefore, reducing the saliency of a region can be understood as performing gamut reduction on it while performing gamut extension in the rest of the image (moving the region away from the gamut boundary). Conversely, increasing the saliency of a region can be understood as performing gamut extension on it while performing gamut reduction in the rest of the image (moving the region closer to the gamut boundary). In this work, we will use the gamut mapping approach of Zamir et al. [17,20], that presents a general framework to perform gamut reduction or gamut extension depending on the value of a particular parameter.

The paper is organized as follows. In the next section we introduce the gamut mapping method of Zamir et al. [17, 20] Then, in section 3, we will explain how to use this gamut mapping method for visual attention retargeting. Section 4 will present the results of our approach. Paper ends in section 5 with the conclusions and further work.

\section{Perceptually-based gamut mapping}

Zamir et al. $[17,20]$ defined an energy functional to perform gamut mapping

$$
\begin{array}{r}
E(I)=\frac{\alpha}{2} \sum_{x}(I(x) \\
-\mu)^{2}+\frac{\beta}{2} \sum_{x}\left(I(x)-I_{0}(x)\right)^{2} \\
-\frac{\gamma}{2} \sum_{x} \sum_{y} w(x, y)|I(x)-I(y)|,
\end{array}
$$

where $\alpha$ and $\beta$ are constant and positive weights, $\gamma$ is a constant and real weight, $I$ is a color channel $(R, G$ or $B), w(x, y)$ is a normalized Gaussian kernel of standard deviation $\sigma, I_{0}$ is the original image, and $\mu$ is the mean average of the original image, and $I(x)$ and $I(y)$ are two intensity levels at pixel locations $x$ and $y$ respectively. This functional is an adaptation from the perceptuallyinspired image energy functional defined by Bertalmío et al. [3] for image enhancement. The resulting evolution equation for the minimization of the above functional can be expressed as

$$
I^{k+1}(x)=\frac{I^{k}(x)+\Delta t\left(\alpha \mu+\beta I_{0}(x)+\frac{\gamma}{2} R_{I^{k}}(x)\right)}{1+\Delta t(\alpha+\beta)}
$$


where the initial condition is $I^{k=0}(x)=I_{0}(x)$. The function $R_{I^{k}}(x)$ indicates the contrast function:

$$
R_{I^{k}}(x)=\frac{\sum_{y \in \mathfrak{I}} w(x, y) s\left(I^{k}(x)-I^{k}(y)\right)}{\sum_{y \in \mathfrak{I}} w(x, y)}
$$

where $x$ is a fixed image pixel and $y$ varies across the image domain $\mathfrak{I}$. The slope function $s(\cdot)$ is a regularized approximation to the sign function, which appears as it is the derivative of the absolute value function in the second term of the functional; in [3] they choose for $s(\cdot)$ a polynomial of degree 7 .

Let us study the energy functional presented in Eq.(1). It presents three competing terms. The first two terms are attachments to the original mean of the image and the original image itself. In contrary, the third term deals with contrast modification. For the gamut mapping problem, the value used for the $\alpha$ parameter in [20] depends on $\gamma: \alpha=\frac{\gamma}{n}$ where $n$ is a natural number. In this way, when considering the value $\gamma=0$ the energy minimization process produces the original image $I_{0}$.

The key point for using this energy functional for both the gamut reduction and the gamut extension problems is the behaviour of the funcional depending on the sign of the $\gamma$ parameter, i.e. the parameter dealing with the contrast modification. Zamir et al. showed that considering a negative value of $\gamma$, the gamut of the image decreases. Furthermore, the smaller the value of $\gamma$, the smaller the gamut of the resulting image. Conversely, if we consider a positive value of the $\gamma$ parameter, the gamut of the image increases. Recently, Zamir et al. [18, 19] also proposed to use for gamut extension the "a" and "b" channels from CIELAB color space instead of the original RGB channels.

\section{Application to visual attention retargeting}

We propose that the high values of saliency in an image are correlated with the degree of color saturation and the proximity to the color gamut boundary. Therefore, our idea is that for reducing the saliency of some regions of an image, we should reduce the gamut of the colors in these regions while we increase the gamut in the rest of the image. Conversely, to increase the saliency of some regions, we should increase the gamut of the colors belonging to these regions while we reduce the gamut of all the other regions.

To perform this idea we define a $\Gamma$ map over the image domain, $\Gamma: \mathbb{D}_{\mathbb{I}}^{2} \longrightarrow \mathbb{R}$. This map will provide us, for each particular pixel $x$ in the image, the value for the parameter $\gamma$ to be used in Eq.(1). Let us note that we will therefore need to reach the steady state of Eq.(2) for each different parameter appearing in the $\Gamma$ map.

Let us now suppose we have a particular region of interest (ROI) $\Upsilon$ in the image in which we want to increase or reduce the saliency. Then, in this paper we propose to use as $\Gamma$ map

$$
\Gamma(x)=\left\{\begin{array}{ccc}
\gamma_{s} & \text { if } & (x) \in \Upsilon \\
-\gamma_{s} & & \text { elsewhere }
\end{array}\right.
$$

where $\gamma_{S}$ will be a positive scalar if we want to increase the saliency of the region of interest and a negative scalar if we want to decrease the saliency. Let us note that, in this particular case, we only need to run to steady state Eq.(2) for two different values.

\section{Results}

To test our approach we use the image dataset presented in [1], which consists of a subset of a larger dataset [7]. The authors of

\begin{tabular}{|c|c|c|}
\hline & Achanta & SIM \\
\hline Original & 0.7907 & 0.7391 \\
\hline Saturation 15\% & 0.8105 & 0.7676 \\
\hline Saturation 25\% & 0.8171 & 0.7816 \\
\hline Ours & $\mathbf{0 . 8 2 3 8}$ & $\mathbf{0 . 7 8 9 1}$ \\
\hline
\end{tabular}

Table 1: Results for the saliency increase case

\begin{tabular}{|c|c|c|}
\hline & Achanta & SIM \\
\hline Original & 0.7907 & 0.7391 \\
\hline Saturation 15\% & 0.7526 & 0.7026 \\
\hline Saturation 25\% & $\mathbf{0 . 7 3 2 3}$ & 0.6845 \\
\hline Ours & 0.7399 & $\mathbf{0 . 6 7 7 3}$ \\
\hline
\end{tabular}

Table 2: Results for the saliency decrease case

this dataset provide us with an image and a mask denoting where the salient object is located.

To show the adequacy of our approach we will consider the mask of the object as our region of interest $\Upsilon$, apply Eq.(4) to obtain the map $\Gamma$, and run our method with those values. When considering a value of $\gamma_{s}$ positive we shall be increasing the saliency of the region of interest, and when considering a value of $\gamma_{s}$ negative, we shall be decreasing it. In particular, we consider two cases for our method: $\gamma_{s}=0.5$ and $\gamma_{s}=-0.5$ for the saliency increase and decrease, respectively. We run our method in RGB color space for gamut reduction and CIELAB color space for gamut extension with the parameters proposed in [20] and [18], respectively. For comparison, we use the most straighforward approach one can probably think of: to reduce (or expand) the saturation inside the region of interest by a percentage and to expand (or reduce) it out of the region by the same percentage (in a similar way to naive gamut mapping methods).

To evaluate the aforementioned approaches we apply two well-known computational saliency methods: Achanta et al. [1] and the SIM method [10]. In order to compute the quantitative results, we make use of a well-known error measure: the area under the ROC curve (AUC). Results are presented in Table 1 and Table 2 for the saliency increase and saliency decrease, respectively. In these tables we can see how our method is able to increase and decrease the saliency of the region of interest for all the three methods and the two error measures. We can also see that our method is outperforming the saturation-based approach in all but one of the cases.

A different question arises at this point. Even if the saliency has been modified properly, do the images look natural? (i.e. can they be shown to an observer?). In Figures 1 and 2 we show the original image (first column), the mask of the region of interest (second column), the results of modifying saturation by $25 \%$ (third column), and our results (fourth column) for the case of saliency increase and saliency decrease, respectively. It is clear that our results, even if they look slightly different from the original image, look natural when are viewed in isolation. In contrary, the results for the saturation modification look unnatural, and therefore can not be considered for applications where a human observer will view the image.

\section{Conclusions}

In this paper we have proposed to use the gamut mapping framework by Zamir et al. [20] to deal with the visual attention 

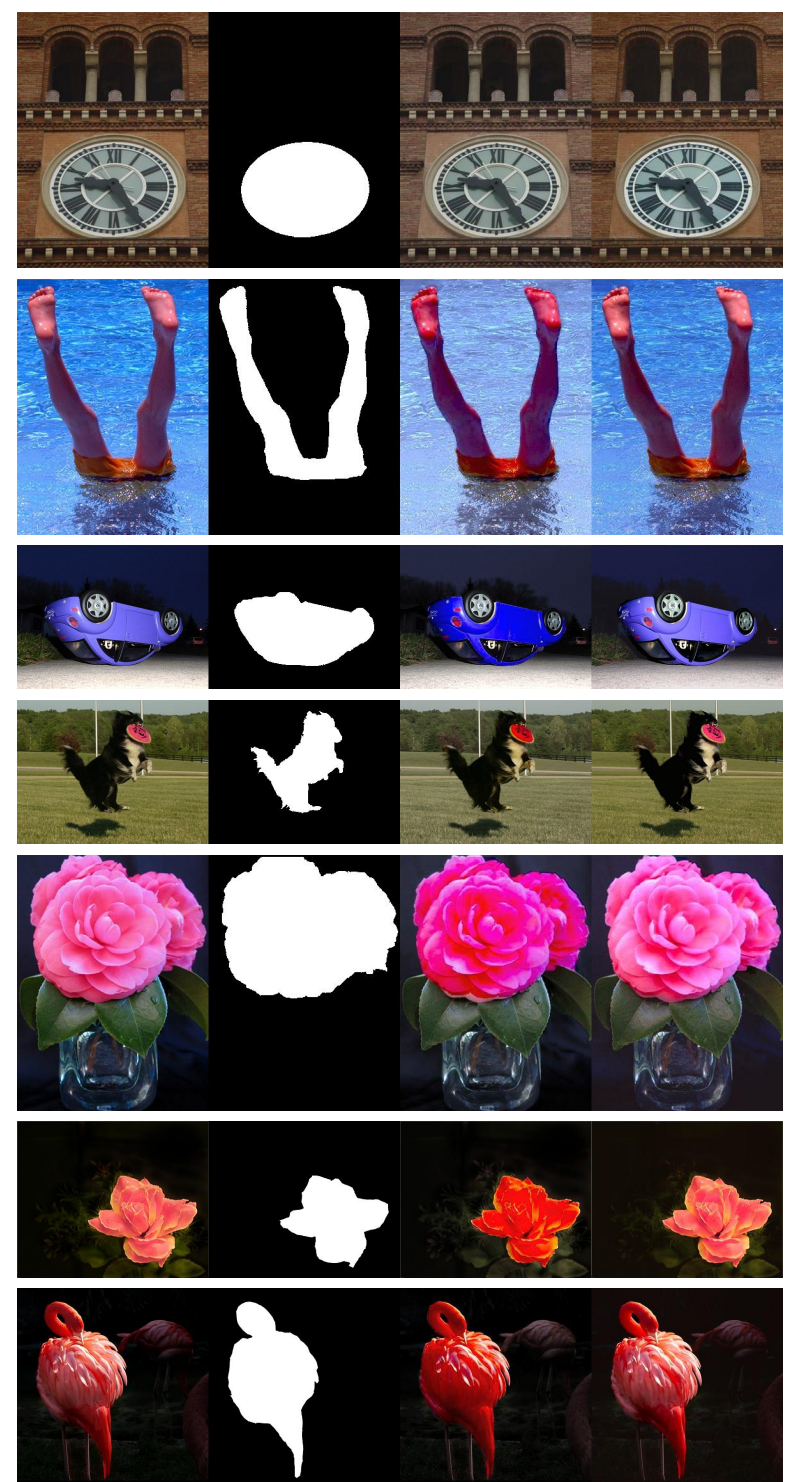

Figure 1. Saliency increase results. From left to right: Original image, region of interest, results of modifying saturation by $25 \%$, our result.

retargeting problem. We have shown that it is possible to decrease the saliency of a region of interest by performing gamut reduction on it and gamut extension on the rest of the image, and to increase the saliency of a region of interest by performing gamut extension on it and gamut reduction on the rest of the image.

Further work might take different directions. First, to study more intricate $\Gamma$ maps in order to, for example, smooth the transitions at the border between the region of interest and the rest of the image. Second, to study the behaviour of our approach under more saliency error measures and more saliency computation methods. Finally, performing thorough psychophysical experiments i) to quantify the subjective preference, naturalness or pleasantness of the processed alternatives and ii) to measure the observers eye fixations to see whether they coincide with the salient areas obtained by the proposed approach.
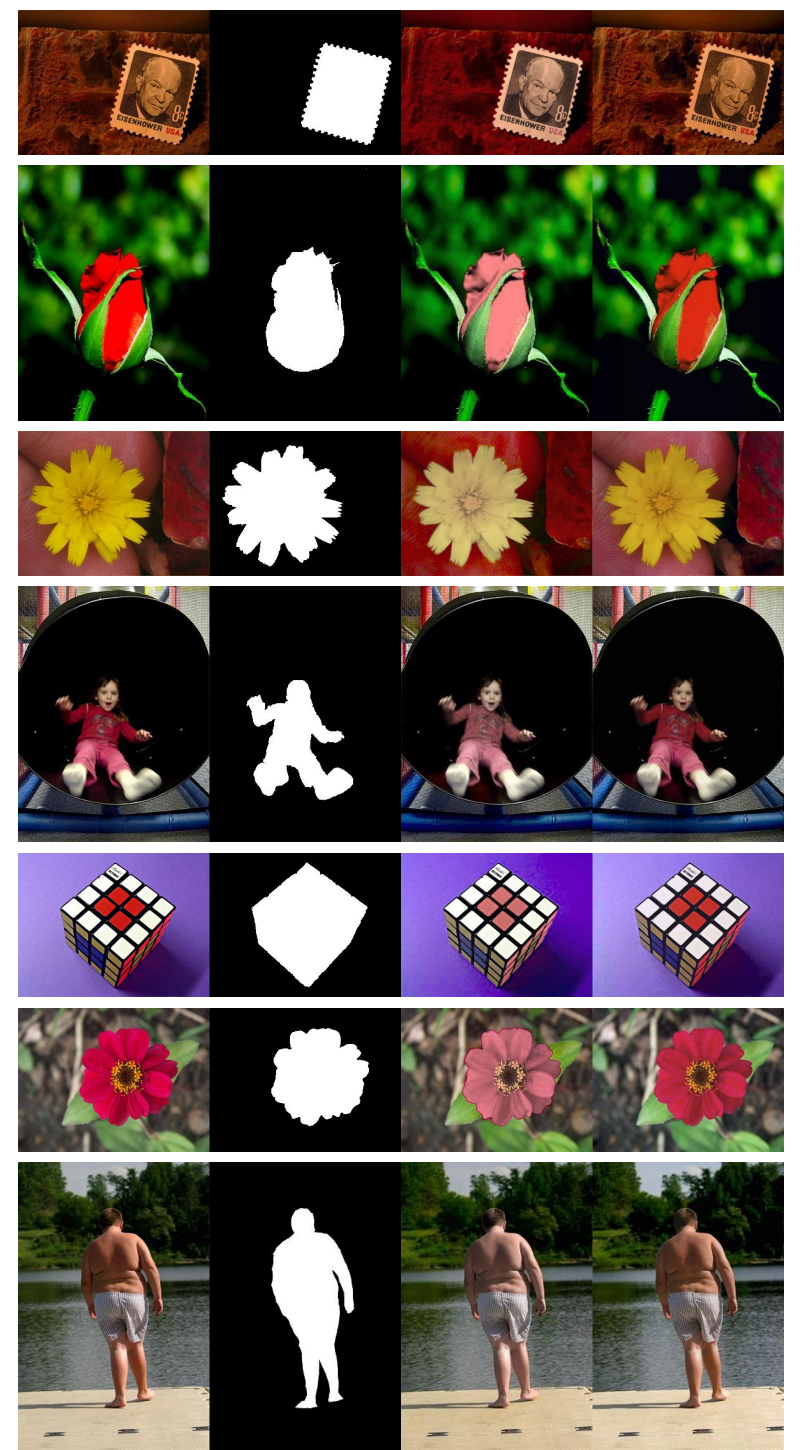

Figure 2. Saliency decrease results. From left to right: Original image, region of interest, results of modifying saturation by $25 \%$, our result.

\section{Acknowledgments}

Authors are supported by the European Research Council, Starting Grant ref. 306337, by the Spanish government FEDER Fund, grant ref. TIN2015-71537-P(MINECO/FEDER,UE), and by the Icrea Academia Award. The work of J. Vazquez-Corral was supported by the Spanish government under Grant IJCI-201419516.

\section{References}

[1] R. Achanta, S. Hemami, F. Estrada, and S. Ss̈strunk. Frequency-tuned Salient Region Detection. In IEEE International Conference on Computer Vision and Pattern Recognition (CVPR 2009), pages 1597 - 1604, 2009.

[2] C. O. Ancuti, C. Ancuti, and P. Bekaert. Enhancing by saliency-guided decolorization. In CVPR, pages 257-264. IEEE, 2011.

[3] M. Bertalmío, V. Caselles, E. Provenzi, and A. Rizzi. Percep- 
tual color correction through variational techniques. IEEE transactions on image processing: a publication of the IEEE Signal Processing Society, 16(4):1058-1072, April 2007.

[4] H.K. Chu, W.H. Hsu, N. J. Mitra, D. Cohen-Or, T.T. Wong, and T.Y. Lee. Camouflage images. ACM Transactions on Graphics, 29(3), 2010.

[5] A. A. Gooch, S. C. Olsen, J. Tumblin, and B. Gooch. Color2gray: salience-preserving color removal. ACM Trans. Graph., 24(3):634-639, 2005.

[6] A. Hagiwara, A. Sugimoto, and K. Kawamoto. Saliencybased image editing for guiding visual attention. In Proceedings of the 1st International Workshop on Pervasive Eye Tracking \&\#38; Mobile Eye-based Interaction, PETMEI '11, pages 43-48, New York, NY, USA, 2011. ACM.

[7] T. Liu, J.Sun, N.N. Zheng, X. Tang, and H.Y. Shum. Learning to detect a salient object. In Computer Vision and Pattern Recognition, 2007. CVPR '07. IEEE Conference on, pages 1-8, June 2007.

[8] V. A. Mateescu and I. V. Bajić. Attention retargeting by color manipulation in images. In Proceedings of the 1st International Workshop on Perception Inspired Video Processing, PIVP '14, pages 15-20, New York, NY, USA, 2014. ACM.

[9] J. Morovič. Color gamut mapping, volume 10. Wiley, 2008.

[10] N. Murray, M. Vanrell, X. Otazu, and C. A. Párraga. Lowlevel spatiochromatic grouping for saliency estimation. IEEE Trans. Pattern Anal. Mach. Intell., 35(11):2810-2816, 2013.

[11] T. V. Nguyen, B. Ni, H. Liu, W. Xia, J. Luo, M. Kankanhalli, and S. Yan. Image re-attentionizing. IEEE Transactions on Multimedia, 15(8):1910-1919, Dec 2013.

[12] M. Pedersen, X. Liu, and I. Farup. Improved simulation of image detail visibility using the non-subsampled contourlet transform. In Color and Imaging Conference (CIC), pages 191-196, Albuquerque, NM, USA, Nov 2013. IS\&T and SID.

[13] S. L. Su, F. Durand, and M. Agrawala. De-emphasis of distracting image regions using texture power maps. In Texture 2005: Proceedings of the 4th IEEE International Workshop on Texture Analysis and Synthesis in conjunction with ICCV'05, pages 119-124, October 2005.

[14] J. K. Tsotsos and N. D. B. Bruce. Saliency based on information maximization. In Y. Weiss, B. Schölkopf, and J. Platt, editors, Advances in Neural Information Processing Systems 18, pages 155-162, MIT Press, 2006. MIT Press.

[15] L. K. Wong and K. L. Low. Saliency retargeting: An approach to enhance image aesthetics. In 2011 IEEE Workshop on Applications of Computer Vision (WACV), pages 73-80, Jan 2011.

[16] Z. Yan, H. Zhang, B. Wang, S.n Paris, and Y. Yu. Automatic photo adjustment using deep neural networks. ACM Trans. Graph., 35(2):11:1-11:15, February 2016.

[17] S. W. Zamir, J. Vazquez-Corral, and M. Bertalmío. Gamut mapping through perceptually-based contrast reduction. In Proc. of Pacific-Rim Symposium on Image and Video Technology, 2013.

[18] S. W. Zamir, J. Vazquez-Corral, and M. Bertalmío. Gamut extension for cinema: Psychophysical evaluation of the state of the art, and a new algorithm. In IS\&T Electronic Imaging Conference, 2015.

[19] S. W. Zamir, J. Vazquez-Corral, and M. Bertalmío. Gamut ex- tension for cinema. IEEE Transactions on Image Processing, 26(4):1595-1606, April 2017.

[20] S.W. Zamir, J. Vazquez-Corral, and M. Bertalmio. Gamut mapping in cinematography through perceptually-based contrast modification. IEEE Journal of Selected Topics in Signal Processing, 8(3):490-503, June 2014.

\section{Author Biography}

Javier Vazquez-Corral received the Ph.D. degree in computer science from the Universitat Autònoma de Barcelona, Bellaterra, Spain, in 2011. He is currently a Juan de la Cierva-Incorporación Research Fellow with the Department of Information and Communication Technologies, Universitat Pompeu Fabra, Barcelona. His research interests are related to the use of color in image processing, computer vision problems, and bridging the gap between color in the human brain and its use in computer-vision applications.

Marcelo Bertalmío was born in Montevideo in 1972. He received the B.Sc. and M.Sc. degrees in electrical engineering from the Universidad de la República, Uruguay, and the Ph.D. degree in electrical and computer engineering from the University of Minnesota in 2001. He is currently an Associate Professor with the Universitat Pompeu Fabra, Spain. He has authored a book titled Image Processing for Cinema. His current research interests are in developing image processing algorithms for cinema that mimic neural and perceptual processes in the human visual system. He was a recipient of the Ramón y Cajal Fellowship, the ICREA Academia Award, and the 2012 SIAG/IS Prize of the Society for Industrial and Applied Mathematics (SIAM) for co-authoring the most relevant image processing work published in the period 2008-2012. He received an ERC Starting Grant for his Project "Image Processing for Enhanced Cinematography" and an ERC Proof of Concept Grant for a tone mapping method. 